\title{
Lebensläufe der Präsidenten und Ehrenmitglieder der DGS
}

Prof. Dr. med. Michael Bamberg

$\nabla$

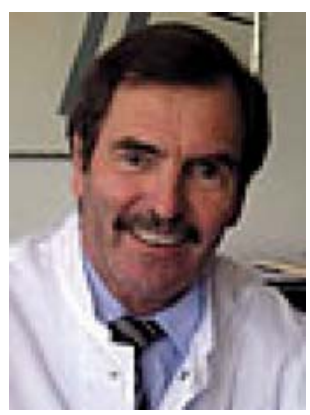

Michael Bamberg wurde im Jahr 1947 in Hamm geboren. Er studierte von 1966 bis 1972 an den Universitäten Bonn, Düsseldorf und Essen. Von 1973 bis 1978 absolvierte er seine Assistenzarztzeit an der Strahlenklinik der Universität Essen, an der Inneren und Chirurgischen Abteilung des MariannenHospitals in Werl und der Röntgenabteilung des Bundeswehrzentralkrankenhauses in Koblenz. Von 1978 bis 1988 arbeitete er als Facharzt, später als Oberarzt für Radiologie und Strahlentherapie an der Strahlenklinik essen, wo er sich auch habilitierte. Seit 1988 ist Bamberg Ärztlicher Direktor der Klinik für Radioonkologie am Universitätsklinikum in Tübingen, von 1991 bis 1992 Dekan der medizinischen Fakultät, von 1995 bis 1997 Sprecher des Interdisziplinären Tumorzentrums Tübingen und seit 1997 Leitender Ärztlicher Direktor des Universitätsklinikums.

Bamberg war im Jahr 1995 Gründungspräsident der Deutschen Gesellschaft für Radioonkologie, von 1998 bis 2000 Stellvertretender Vorsitzender im Verband der Universitätsklinika Deutschlands, von 2004 bis 2008 Präsident der Deutschen Krebsgesellschaft.

Er ist Vorstandsvorsitzender der Deutschen Krebsstiftung und Gründungsmitglied des Nationalen Krebsplans. Er ist Verfasser zahlreicher Bücher und Fachpublikationen, war und ist Vorstandsmitglied mehrerer internationaler Fachgesellschaften, seit 1999 Mitglied der Deutschen Akademie der Naturforscher Leopoldina. Er hat zahlreiche Ehrungen und Preise entgegengenommen und ist Ehrenmitglied zahlreicher Deutscher und ausländischer Fachgesellschaften. Im Jahr 2008 wurde Bamberg zum Ehrenmitglied der Deutschen Gesellschaft für Senologie ernannt.

\section{Prof. em. Dr. med. Roland Bässler}

$\nabla$

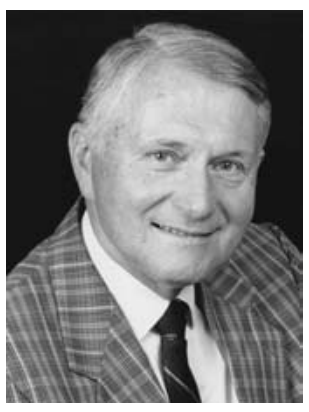

Roland Bässler wurde im Jahr 1926 im sächsischen Niederwiesa geboren. Im ersten Nachkriegssemester 1945/46 in Jena holte er nach seiner Verpflichtung beim Arbeitsdienst und bei der Wehrmacht das Abitur nach und begann das Medizinstudium. Nach dem Physikum 1948 wechselte Bässler zur Universität Leipzig. Seine Assistenzarztzeit absolvierte er in Bad Berka/ Thüringen am Bergbaukrankenhaus Stollberg/Erzgebirge, am Anatomischen und am Pathologischen Institut der Universität Leipzig und ab 1956 am PathologischBakteriologischen Institut in Hannover, wo er sich in die Elektronenmikroskopie einarbeiten konnte. 1959 wechselte Bässler nach Mainz, nachdem Prof. Dr. H. Bredt, sein früherer Chef in Leipzig, den Ruf und das Direktoriat des Pathologischen Instituts der Universität Mainz angenommen hatte. In Mainz erfolgte 1963 die Habilitation, 1969 die Ernennung zum apl. Professor und 1970 zum Professor und Wissenschaftlichen Rat. 1972 übernahm Bässler die Chefarztstelle am Pathologischen Institut des Klinikums Fulda, die er bis 1993 bekleidete.

Das in Fulda 1976 gegründete „Mamma-Register“ für konsiliarische Untersuchungen betreute Bässler bis 1999. Es wurden ca. 7000 Problemfälle der Mamma-Pathologie untersucht und die Ergebnisse 2001 ausgewertet und publiziert. Sein wissenschaftliches Interesse an Fragen der Mammapathologie führte zu zahlreichen Publikationen, zur Mitarbeit an zahlreichen Referenzwerken und zur Monografie „Pathologie der Brustdrüse“ 1978. 1983 war Bässler Tagungspräsident der Deutschen Gesellschaft für Senologie, im Jahr 1985 Kongresspräsident und Vorsitzender der Deutschen Gesellschaft für Pathologie.

Bässler wurde mit zahlreichen Preisen und Ehrungen ausgezeichnet, darunter der Preis der Deutschen Krebshilfe, die Ehrenmitgliedschaft der Internationalen Akademie für Pathologie, die der Deutschen Gesellschaft für Senologie und die Rudolf-Virchow-Medaille der Deutschen Gesellschaft für Pathologie.

\section{Prof. em. Dr. med. Heinrich Maass}

$\nabla$

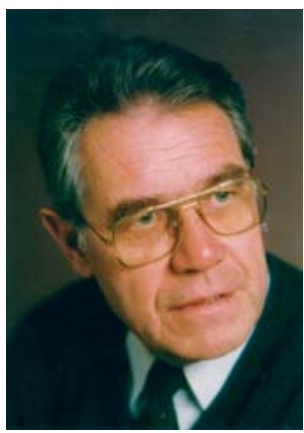

Heinrich Maass wurde im Jahr 1927 in Flensburg geboren. Sein Medizinstudium absolvierte er zwischen 1948 und 1953 in Hamburg, Zürich und Düsseldorf. Anschließend arbeitete er als Stipendiat der Deutschen Forschungsgemeinschaft am UFK Hamburg und am Biochemischen Institut. Zwischen 1956 und 1968 war er als wissenschaftlicher Assistent und später als Oberarzt an der UFK Hamburg-Eppendorf tätig und erhielt dort eine apl. Professur. Zwischen 1976 und 1984 war er Chefarzt an der Frauenklinik des Zentralkrankenhauses Bremen. 1984 trat er als Direktor der UFK Hamburg Eppendorf die Nachfolge von Prof. Klaus Thomsen an und blieb auf dieser Position bis zu seiner Emeritierung im Jahr 1995.

Das wissenschaftliche Interesse von Heinrich Maass galt immer der Krebsbekämpfung, vor allem dem Brustkrebs. Er führte das Hormonrezeptor-Konzept zur Therapieselektion in Europa ein, ebenso wird die Planung und Durchführung großer klinischer Studien zur Therapie des Mammakarzinoms.

Maass war von1987 bis 1990 Präsident der Deutschen Gesellschaft für Senologie und von 1990 bis 1992 Präsident der Deutschen Krebsgesellschaft. Er wurde für sein lebenslanges Engagement in der Krebsbekämpfung mit zahlreichen Preisen geehrt, darunter der Wilhelm-Warner-Preis, die Karl-Heinrich-BauerMedaille der Deutschen Krebsgesellschaft und die Carl-Kauf- 
mann-Medaille der Deutschen Gesellschaft für Gynäkologie und Geburtshilfe. Heinrich Maass ist Ehrenmitglied der Deutschen Gesellschaft für Senologie.

\section{Prof. Dr. med. Hans-Joachim Frischbier}

$\nabla$

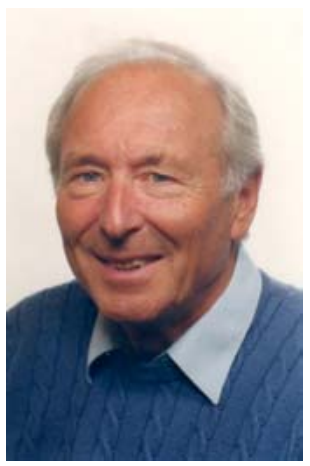

Hans-Joachim Frischbier wurde im Jahr 1932 in Berlin geboren. Er studierte Medizin in Hamburg, wo er im Jahr 1958 an der UFK Hamburg Eppendorf mit der ärztlichen Weiterbildung begann. Im Jahr 1959 wechselte Frischbier nach Heidelberg, wo in der Brustkrebs-Diagnostik bereits eine enge Zusammenarbeit mit dem Straßburger Radiologen Prof. Ch.M.Gros bestand, dem späteren Gründer der Europäischen Gesellschaft für Senologie

(1972). Diese Zusammenarbeit wurde prägend für die weitere Arbeit von Hans-Joachim Frischbier, der im Jahr 1964 zunächst als Facharzt für Röntgenologie und Strahlenkunde und 1966 als Facharzt für Frauenheilkunde und Geburtshilfe anerkannt wurde und sich im gleichen Jahr - zurück in Hamburg - habilitierte. 1973 wurde Frischbier zum Direktor der Abteilung für Gynäkologische Radiologie und zum Professor ernannt. Die Abteilung leitete er bis zu seinem Ruhestand im Jahr 1996.

Ab 1971 begann Frischbier in seiner Klinik mit MammografieReihenuntersuchungen im Rahmen einer gynäkologischen Krebs-Vorsorgeuntersuchung ohne klinische verdächtigen Tastbefund in zweijährlichen Kontrollabständen. Die Ergebnisse 52 klinisch unauffällige Tumoren bei 11450 Frauen - deckten sich mit der internationalen Literatur. Nach dem bahnbrechenden Kongress von Ch. M. Gros in Strasburg 1972 über die „Nichtverstümmelnden Therapiemöglichkeiten der malignen Mammatumoren“ begann das Team um Frischbier unter Leitung von Prof. K.Thomsen, eigene Erfahrungen mit der Strahlentherapie bei speziellen Fällen unter Vermeidung einer Mastektomie zu sammeln. Über die erfolgversprechenden Ergebnisse, die von der Fachwelt mehr als kritisch kommentiert wurden, berichteten die Ärzte auf vielen Tagungen.

Im Jahr 1978 wurde Frischbier zum Vizepräsidenten der Internationalen Gesellschaft für Senologie ernannt und erhielt den Auftrag, den ersten internationalen Kongress der Fachgesellschaft in Deutschland auszurichten. Dieer Kongress fand im Jahr 1980 in Hamburg statt. Über 1000 Kolleginnen und Kollegen aus allen Teilen der Welt und aus allen senologischen Fachgebieten nahmen teil. Auf diesem Kongress wurde die Idee geboren, eine Deutsche Gesellschaft für Senologie zu gründen. Der erste Kongress der neuen Fachgesellschaft fand im März 1981 in Heidelberg unter Leitung von Prof. Kubli statt. Bis 1986 war Frischbier Vorsitzender. Im Jahr 1996 wurde er zum Ehrenmitglied ernannt. 1998 wurde Frischbier zum Ehrenpräsidenten der Internationalen Gesellschaft für Senologie ernannt.

Gemeinsam mit seiner langjährigen Oberärztin Dr. Ingrid Schreer, mit Prof. Hoeffken, Köln und Prof. Robra, Hannover startete Frischbier in Abstimmung mit dem Bundesausschuss Ärzte und Krankenkassen die „Deutsche Mammographie-Studie“, die letztlich die Grundlage für das heutige Mammografiescreening wurde.

\section{Prof. Dr. med. Walter Hoeffken}

$\nabla$

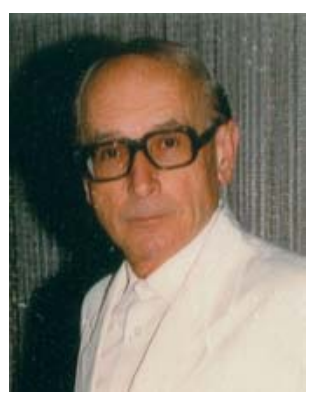

Walter Hoeffken wurde im Jahr 1917 in Köln geboren. Nach dem Abitur 1937 studierte er Medizin in Innsbruck, Marburg, Köln und Prag, immer wieder unterbrochen durch Einberufungen in den Militärdienst, zuletzt als Sanitätsoffizier. Im Jahr 1944 erfolgten in Prag Approbation und Promotion und anschließend weiterer Einsatz in Lazaretten, nach Kriegsende die Leitung eines Hilfskrankenhauses an einem Flüchtlingslager des Regierungsbezirks Köln.

Von 1947 bis 1955 erhielt Hoeffken seine radiologische Ausbildung am Röntgeninstitut des Krankenhauses Köln-Merheim. Ab 1955 leitete Hoeffken die Städtische Röntgenabteilung des Bürgerhospitals Köln, das im Jahr 1958 dem Universitätsklinikum angegliedert wurde. 1957 erfolgte die Habilitation, 1963 die Ernennung zum apl. Professor. Bis 1966 leitete er die Röntgenabteilung der Medizinischen und Chirurgischen Universitätskliniken und betreute zudem die Universitäts-Kinderklinik. Danach übernahm Hoeffken die Position als Chefarzt am Strahleninstitut der AOK Köln mit Angiologie-Abteilung, Mammografie-Abteilung und einem weiten diagnostischen und therapeutischen Spektrum.

Hoeffken ist Autor zahlreicher wichtiger Publikationen und Standardwerke, vor allem zur Röntgenuntersuchung der weiblichen Brust. Im Jahr 1980 war er Präsident des Deutschen Röntgenkongresses, 1982 Präsident der wissenschaftlichen Tagung der Deutschen Gesellschaft für Senologie, 1987 bis 1993 Leiter der Deutschen Mammographie-Studie des BMFT, 1989 bis 1993 Vorsitzender der Deutschen Gesellschaft für Senologie.

Hoeffken war im Vorstand oder Beirat zahlreicher Fachgesellschaften und Institutionen, darunter der Deutschen Krebshilfe und der Wilhelm-Vaillant-Stiftung.

Hoeffken verstarb im Jahr 1999 im Alter von 82 Jahren.

\section{Gabriel N. Hortobagyi, MD, FACP}

$\nabla$

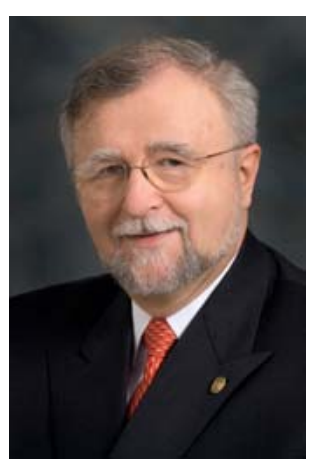

Gabriel N. Hortobagyi, MD, FACP, wurde im Jahr 1945 in Budapest in Ungarn geboren. Der Arztberuf war seit früher Kindheit sein Ziel. Seine Familie war in der Zeit des Kalten Krieges erheblichen Repressalien ausgesetzt, er selbst und seine Schwester durften keinen höheren Schulabschluss machen. Die Familie emigrierte später nach Kolumbien, wo Hortobagyi im Jahr 1970 an der Universität von Bogota sein Medizinstudium beenden konnte. Seine Ausbildung in Innerer Medizin und Onkologie schloss er im St.Luke's Hospital in Cleveland, Ohio, and im Anderson Cancer Center in Houston, Texas, ab. Sein Forschungsschwerpunkt sind Biologie und Management des Brustkrebs. Fast 1000 eigene Publikationen belegen sein exzeptionelles Engagement.

Hortobagyi ist ein Pionier der multidisziplinären Brustkrebs-Therapie. Er entwickelte das Konzept der Kombinationstherapien für inoperable Brusttumoren, führte die präoperative Chemotherapie zur Reduktion der Tumorgröße bei Patientinnen ein, bei denen der 
Primärtumor bereits Fernmetastasen gesetzt hat, Er kombinierte Chirurgie, Radiatio und Chemotherapie und führte zahlreiche bahnbrechende Studien durch. Seine Untersuchungen werden seit 1993 von der Breast Cancer Research Foundation unterstützt.

Von 2006 bis 2007 war er Präsident der American Society of Clinical Oncology, von 1996 bis 1998 Präsident der Société Internationale de Sénologie (SIS) und ist Ehrenmitglied der Deutschen Gesellschaft für Senologie.

Er wurde international vielfach hoch geehrt und wurde mit zahlreichen Preisen, Ehrenmitgliedschaften und Ehrenpräsidentschaften ausgezeichnet, darunter der Orden der Ehrenlegion (Frankreich).

\section{Prof. Dr. med. Walter Jonat}

$\nabla$

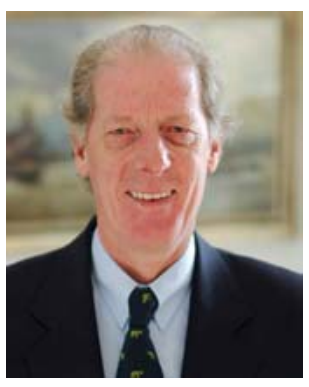

Walter Jonat wurde 1949 in Hamburg geboren. Sein wissenschaftlicher Werdegang führte ihn über Stationen in Hamburg und Bremen, an die Universität Hamburg und von dort nach Kiel. Dort ist er seit 1995 Direktor der Klinik für Gynäkologie und Geburtshilfe des Universitätsklinikums Schleswig-Holstein, Campus Kiel. Der Schwerpunkt seiner klinischen und wissenschaftlichen Tätigkeit liegt auf der Diagnostik und Therapie des Mammakarzinoms.

Er ist Mitglied verschiedener nationaler und internationaler wissenschaftlicher Gesellschaften, unter anderem der deutschen Krebsgesellschaft (DKG), der American Society of Clinical Oncology (ASCO) und der American Association of Cancer Research (AACR). 2008 wurde er ehrenhalber als Fellow in das Royal College of Obstetrics and Gynecology (UK) aufgenommen. 20062008 war er Präsident der Deutschen Gesellschaft für Gynäkologie (DGGG). Aktuell ist er Executivmitglied der Fédération Internationale de Gynécologie et d'Obstétrique (FIGO), sowie Mitglied des erweiterten Vorstandes der Deutschen Krebshilfe (DKH). Seit 2002 ist Walter Jonat Mitglied des wissenschaftlichen Beirats der Deutschen Gesellschaft für Senologie. 2005 war er Kongresspräsident der 25. Jahrestagung der Gesellschaft in Stuttgart. Im Jahr 2010 wurde er zum Ehrenmitglied der Deutschen Gesellschaft für Senologie ernannt.

Jonat wurde mehrfach für seine herausragende wissenschaftliche Arbeit ausgezeichnet. Er erhielt unter anderem den Staude-Plannenstiel-Award, den Deutschen Krebspreis, den Wilhelm Warner Preis, den Gunther-Bastert-Preis und den Kubli-Preis.

\section{Prof. Dr. med. Rolf Kreienberg}

$\nabla$

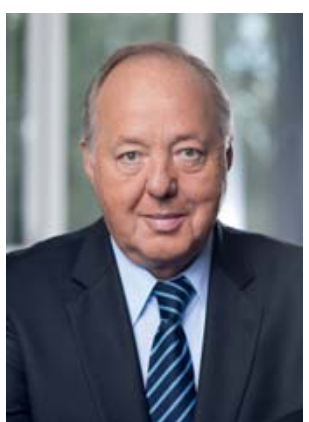

Rolf Kreienberg wurde im Jahr 1946 in Kaiserslautern geboren. Sein wissenschaftlicher Werdegang führte ihn über eine immunologische Grundausbildung zur Frauenklinik am Universitätsklinikum Mainz, zu deren kommissarischer Direktor er im Jahr 1988 ernannt wurde. 1992 folgte er dem Ruf der Universität Ulm auf den Lehrstuhl für Gynäkologie. Seitdem ist er Direktor der Ulmer Universitäts-Frauenklinik.

Sein wissenschaftlicher Schwerpunkt ist die Onkologie, insbesondere das Mammakarzinom. Seit 2004 ist Kreienberg feder- führender Koordinator der S3-Leitlinie „Diagnostik, Therapie und Nachsorge des Mammakarzinoms“ und Leitlinienbeauftragter der Deutschen Gesellschaft für Gynäkologie und Geburtshilfe. Unter seiner Leitung wurde das Studiennetzwerk BRENDA (Breast Cancer Care under Evidence based Guidelines), dessen Untersuchungsergebnisse maßgeblich dazu beitragen, die Versorgungsqualität bei Patientinnen mit Brustkrebs zu verbessern. Für seine Verdienste um eine optimierte Vorsorge und Therapie bei Brustkrebserkrankungen wurde Kreienberg im Jahr 2010 mit der Johann-Georg-Zimmermann-Medaille ausgezeichnet.

Kreienberg war von 2000 bis 2002 Präsident der Deutschen Krebsgesellschaft und von 2008 bis 2010 Präsident der Deutschen Gesellschaft für Gynäkologie und Geburtshilfe. Auf der 31.Jahrestagung der Deutschen Gesellschaft für Senologie wurde Kreienberg zum Ehrenmitglied der Fachgesellschaft gewählt.

\section{Prof. Dr. med. Rolf Sauer}

$\nabla$

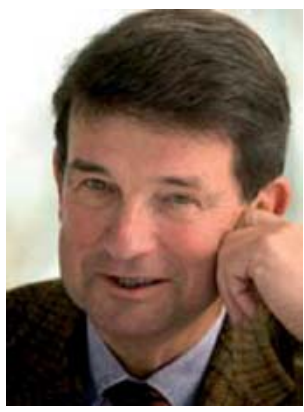

Rolf Sauer wurde 1939 in Hamburg geboren. Er wuchs in Naumburg/Saale in der ehemaligen DDR auf. Sein wissenschaftlicher Werdegang führte ihn über die Universitäten Hamburg, Wien, Basel, Erlangen. 1977 übernahm er am Universitätsklinikum Erlangen den ersten Lehrstuhl für Strahlentherapie in Bayern. Über 30 Jahre lang war Prof. Sauer Direktor der Radioonkologie an diesem Klinikum, später Direktor des Klinikums, und prägte das Fach maßgeblich. Sauer leitete eine Vielzahl von klinischen Studien und setzte sich früh für brusterhaltende Therapiekonzepte beim Mammakarzinom ein. Es darf als sein Verdienst bezeichnet werden, dass in den 80erJahren der Brusterhalt beim Mammakarzinom flächendeckend in Deutschland eingeführt wurde. Die zahlreichen Ämter, die er bekleidet hat, spiegeln seine intensive interdisziplinäre Tätigkeit: Vorsitz in der AG Radiologische Onkologie in der Deutschen Krebsgesellschaft, Vorstandsmitglied der Deutschen Röntgengesellschaft und Vorsitzender der Deutschen Gesellschaft für Radiologische Onkologie (DEGRO). Im Jahr 2000 wurde er zum Vorstandsmitglied in der Deutschen Gesellschaft für Senologie ernannt undwar Kongresspräsident der DGS im Jahr 2002.

Sauer wurde mit zahlreichen Preisen und Ehrenmitgliedschaften ausgezeichnet, so erhielt er als erster Strahlentherapeut den Deutschen Krebspreis. Im Jahr 2009 verlieh ihm die Deutsche Gesellschaft für Senologie die Ehrenmitgliedschaft.

\section{Prof. Dr. med. Ingrid Schreer}

$\nabla$

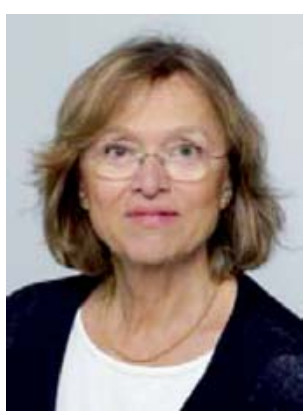

Ingrid Schreer wurde 1944 geboren. Sie studierte Medizin an der Universität Freiburg, wo sie auch die Weiterbildung zur Fachärztin für Radiologie absolvierte. Anschließend wechselte sie als Oberärztin in die Abteilung für Gynäkologische Radiologie der UniversitätsFrauenklinik Hamburg-Eppendorf. In Hamburg absolvierte sie zusätzliche eine Ausbildung zur Psychotherapeutin. 1993 habilitierte sie für das Fach Gynäkologische Radiologie. Von 1998 bis 2010 war sie Leitende Oberärztin des Mammazentrums des Universitätsklinikums Kiel. 
Schreer setzte sich seit den 90er Jahren intensiv für eine breit angelegte Brustkrebs-Früherkennung ein. Sie führte in Schleswig Holstein die „Qualitätsgesicherte Mamma-Diagnostik“ (QuaMaDi) ein mit höchsten Anforderungen an die Qualität der radiologischen Diagnostik. Dass seit 2007 jede Frau in Deutschland, die zwischen 50 und 59Jahren alt ist, alle zwei Jahre an einem hochwertigen Screening auf Mammakarzinom teilnehmen kann, ist unter anderem ihrem Einsatz zu verdanken. Ihre Leistungen auf dem Gebiet der Brustkrebs-Früherkennung wurden 2003 mit dem Bundesverdienstkreuz am Bande ausgezeichnet.

Von 2004 bis 2006 war Schreer Präsidentin der European Society of Breast Imaging. Von 199 bis 2003 war sie Präsidentin der Deutschen Gesellschaft für Senologie, im Jahr 2010 Kongresspräsidentin des DGS-Fortbildungskongresses.

Im Jahr 2009 wurde Schreer zur Ehrenpräsidentin der Deutschen Gesellschaft für Senologie ernannt.

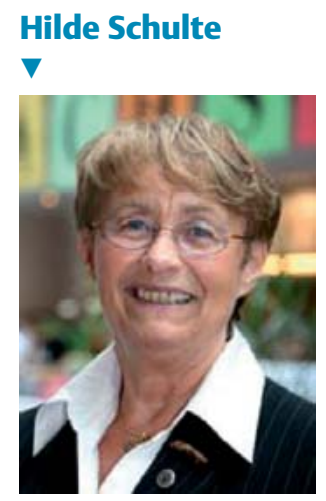

Hilde Schulte wurde 1941 geboren. Sie war viele Jahre Heimleiterin in Hannover, als sie mit 48Jahren an Brustkrebs erkrankte. In der Zeit der Krankheit und der Rekonvaleszenz -damals ohne multidisziplinäres ärztliches Betreuungskonzept und ohne Selbsthilfeorganisation - entwickelte sie die Fähigkeit, ihre eigenen Bedürfnisse wahrzunehmen und Hilfe und Unterstützung anzunehmen. Im Kontakt mit anderen Brustkrebspatientinnen wurde ihr klar, wie hilfreich Austausch und Ermutigung von gleichermaßen Betroffenen sein konnte. Sie nahm Kontakt zu den regionalen Anlaufstellen der Frauenselbsthilfe nach Krebs e.V.(FSH) auf und wurde bereits wenige Jahre später zur stellvertretenden Vorsitzenden in den Landesvorstand Hessen gewählt, 1999 als Vorstandsmitglied in den Bundesvorstand. 2001 wurde sie zur Vorsitzenden des Bundesverbandes gewählt.

Bis zu dieser Zeit lag der Arbeitsschwerpunkt der FSH auf dem Netzwerken und dem Gespräch mit betroffenen Frauen. Hilde Schulte begann, Kontakte in die Gesundheitspolitik, die Wissenschaft, zu Vertretern von Krankenkassen und von anderen Selbsthilfeorganisationen zu knüpfen. Von großer Bedeutung war das Zusammentreffen von Hilde Schulte mit Prof. K.-D.Schulz, zu dieser Zeit Präsident der Deutschen Gesellschaft für Senologie. Sie wurde als Vertreterin der Selbsthilfe in das Autorenteam der beiden S3-Leitlinien „Brustkrebs-Früherkennung“ und „Diagnostik, Therapie und Nachsorge des Mammakarzinoms“ aufgenommen und erarbeitete sich und der Frauenselbsthilfe in der Gesundheitspolitik Achtung und Ansehen.

„Dass die Krebs-Selbsthilfe bei der Ärzteschaft und in der Gesundheitspolitik heute eine so hohe Akzeptanz genießt, ist maßgeblich ein Verdienst von Hilde Schulte. Auf glaubwürdige, kompetente und hartnäckige Art hat sie alle Beteiligten im Versorgungssystem von der Notwendigkeit ihrer Anliegen überzeugt und der Patientenbeteiligung Tür und Tor geöffnet. Hilde Schulte hat damit einen unschätzbaren Dienst für viele Krebspatienten geleistet.“ (Gerd Nettekoven, Deutsche Krebshilfe, 2010)

\section{Prof. em. Dr. med. Klaus-Dieter Schulz}

$\nabla$

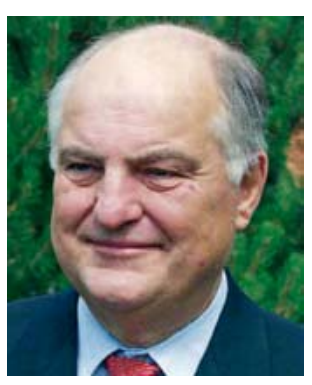

Klaus Dieter Schulz wurde im Jahr 1946 geboren. Seine medizinische Ausbildung begann er an der UniversitätsFrauenklinik in Hamburg im Jahr 1962. Er habilitierte bei G. Bettendorf über den Wirkmechanismus von Clomiphen, überprüfte die Wirkung von Röntgenstrahlen auf den DNA-Synthesezyklus und wies nach, dass der Effekt zyklusabhängig ist. Für diese Arbeiten erhielt er gemeinsam mit H. Maass bereits im Jahr 1967 den MartiniPreis der Bundesärztekammer. 1981 bekam er den Ruf auf den Lehrstuhl für Gynäkologie, gynäkologische Endokrinologie und Onkologie in Marburg.

Über 20Jahr lang beeinflusste Klaus-Dieter Schulz die Entwicklung in der Onkologie maßgeblich, setzte sich hier vor allem für die Früherkennung ein und brachte die erste S3-Leitlinie zu diesem Thema auf den Weg, die im Jahr 2003 von der Deutschen Gesellschaft für Senologie unter seiner Federführung erstmals publiziert wurde und dauerhafte Maßstäbe für jedes Früherkennungsprogramm in Deutschland definiert hat.

Schulz hat entscheidende Impulse für die Versorgungsforschung gegeben, war wichtiger Gesprächspartner in der Gesundheitspolitik ebenso wie in der Frauenselbsthilfe nach Krebs. Er war Präsident der World Society for Breast Health, Präsident der Deutschen Gesellschaft für Senologie, ist Ehrenmitglied der Deutschen Gesellschaft für Gynäkologie und Geburtshilfe, der Deutschen Gesellschaft für Senologie und der Österreichischen Gesellschaft für Senologie.

Schulz verstarb plötzlich und unerwartet im Jahr 2007. Die Senologie achtet ihn als einen ihrer wichtigsten Vertreter in Wissenschaft und Gesundheitspolitik im In- und Ausland.

\section{Hofrat Prof. em. Dr. med. Alfred Staffen}

$\nabla$

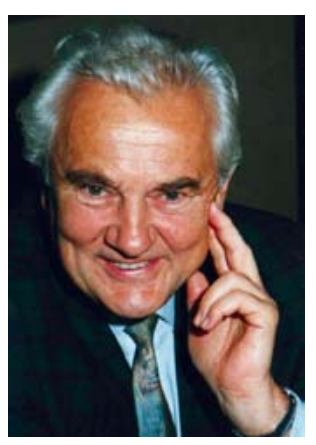

Alfred Staffen wurde im Jahr 1935 in Wien geboren. Seine medizinische Ausbildung begann er im Jahr 1960 an der Chirurgischen Universitätsklinik Wien, wo er sich 1980 habilitierte. Seit Anfang der 70er-Jahre beschäftigte sich Staffen intensiv mit der stadiengerechten chirurgischen Versorgung des Mammakarzinoms, gemeinsam mit der strahlentherapeutischen Universitätsklinik Wien, wodurch es möglich war, das brusterhaltende Konzept der Brustkrebstherapie schon früh in Österreich einzuführen.

1983 wurde Staffen zum Hofrath ernannt, 1987 zum Universitätsprofessor für Chirurgie in Wien, 1993 zum Stellvertretenden Leiter der Universitätsklinik für Herz-Thorax-Chirurgie.

Staffen war Gründungsmitglied der Österreichischen Gesellschaft für Senologie, Präsident dieser Fachgesellschaft von 1992 bis 1993 und Copräsident des 2.Europäischen Kongresses für Senologie in Wien im Jahr 1994. Von 1990 bis 2004 war er Vorstandsmitglied der Deutschen Gesellschaft für Senologie als Vertreter Österreichs und hat sich in diesen Jahren gemeinsam mit 
Klaus Dieter Schulz um die Zusammenarbeit der drei deutschsprachigen Gesellschaften für Senologie verdient gemacht. Im Jahr 1998 wurde er zum Ehrenmitglied der Deutschen Gesellschaft für Senologie ernannt.

\section{Prof. Dr. med. Umberto Veronesi}

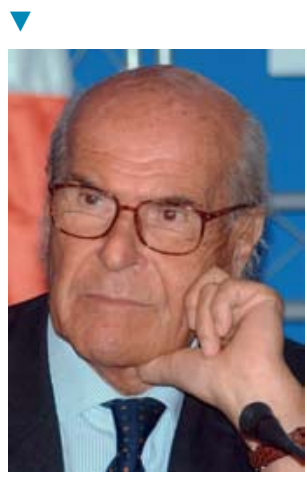

Umberto Veronesi wurde im Jahr 1925 in Mailand geboren und studierte an der dortigen Universität Medizin. Von 1975 bis 1994 war er Direktor des italienischen Nationalen Krebsinstituts. 1982 stand er als Präsident der Internationalen Union gegen Krebs, UICC, vor und gründete die „European School of Oncology“ in Mailand, deren wissenschaftlicher Direktor er ist. Von 2000 bis 2001 war er italienischer Gesundheitsminister.
Ein Schwerpunkt seiner Arbeit ist die Weiterbildung aller im senologischen Bereich tätigen Therapeuten. Die von ihm gegründete „European School of Oncology“ ist heute ein Maßstab in ganz Europa und wurde zum Beratungsorgan für die Europäische Gemeinschaft. Die „European Society of Surgical Oncology“, ebenfalls von ihm gegründet, führt diesen Weg der Weiter- und Fortbildung fort.

Bereits 1968 schlug Veronesi auf einem WHO-Symposium eine Studie zu brusterhaltenden Operationstechniken vor. 1972 begann er, die ersten Frauen innerhalb dieser Studie brusterhaltend zu operieren. 1981 publizierte er seine Ergebnisse im New England Journal of Medicine - ein internationaler Meilenstein für die brusterhaltende Therapie des Mammakarzinoms.

Weitere Schwerpunkte seiner Arbeit sind die Themen rund um den Sentinel-Node und die Sekundärprävention.

Veronesi ist Ehrendoktor an zahlreichen namhaften Universitäten. Er ist Ehrenpräsident der Senologic International Society (SIS) und Ehrenmitglied der Deutschen Gesellschaft für Senologie. 